\title{
A HYBRID MODEL TO PREDICT LOCALISED CRACKS OF REINFORCED CONCRETE SLABS IN FIRE
}

\author{
Feiyu Liao, Zhaohui Huang \\ Department of Mechanical, Aerospace and Civil Engineering, Brunel University, Uxbridge, Middlesex UB8 3PH, UK
}

\begin{abstract}
This paper presents a robust hybrid finite element procedure for predicting the large individual cracks within reinforced concrete floor slabs at elevated temperatures. For modelling the cracks formations and propagations within the floor slabs, the smeared crack model is used for modelling early stages of crack evolution, and then the 'delayed extended finite element method (D-XFEM) is proposed for capturing individual big cracks within the floor slabs. The new model has been validated against previous fire test results. A series of parametric studies has been conducted on a composite floor to understand the influences of different protection conditions of the support steel beams on both global responses and cracking patterns of the composite floor under fire conditions.
\end{abstract}

Keywords: Integrity failure, reinforced concrete slabs in fire, smeared crack, extended finite element method.

\section{INTRODUCTION}

Previous research indicates that under fire conditions, the behaviours of steel-framed composite structure are significantly influenced by the concrete floor (Bailey and Toh, 2007; Huang et al., 2003). However, reinforced concrete slabs are often forced into large deformation in fire, resulting in the formation of large individual cracks within the floor slabs. This phenomenon has been observed in some previous experimental tests (Bailey and Toh, 2007). Those large individual cracks could cause severe integrity failure within the floor slabs, and fire would even spread from lower floor to upper floor through such big cracks' opening. Therefore, predicting the opening of individual large cracks within the floor slabs can be a crucial issue to assess the fire resistance of floor slabs as well as fire safety of the whole frame system.

In the past, the smeared crack model and discrete-cracking model were traditionally used for modelling concrete cracking. However, the smeared cracking model is not able to capture the opening of individual cracks within the concrete members. For the discrete-cracking model, limiting the cracks to inter-element boundaries may cause mesh bias, and requires performing costly re-meshing during the analysis process. In order to overcome the re-meshing process, the extended finite element method (XFEM) was introduced (Moës et al., 1999) based on the partition of unity theory. Recently, XFEM formulation was also applied in yield line analyses of concrete slabs (Xu et al., 2013). However, all XFEM models described above for modelling plate/shell elements were all restricted to an initial through-the-thickness crack within plate or shell structures. They are not capable of predicting the individual cracks formation and propagations within the reinforced concrete floor slabs. Moreover, in these previous models, the influence of temperatures was not included. So far, very limited efforts have been made to use the XFEM in modelling reinforced concrete or composite floor slabs under fire conditions.

The main objective of this paper is to develop a nonlinear finite element procedure to predict individual large cracks within the reinforced concrete slabs in fire. In this new model, the reinforced concrete slabs are modelled as an assembly of plain concrete plate, reinforcing steel bar and bondlink elements. The plain concrete plate elements are sub-divided into layers to take into account the temperature distribution over the cross-section of the slab. A robust hybrid finite element procedure has been developed for capturing individual big cracks within the reinforced concrete slabs under fire conditions. 


\section{DEVELOPMENT OF NONLINEAR PROCEDURE}

As shown in Fig. 1 (a), a reinforced concrete slab is modelled as an assembly of plain concrete, reinforcing steel bar, and bond-link elements. The plain concrete is represented as quadrilateral nine-node higher-order isoparametric thick plate elements (Huang et al., 2003) and both geometric and material nonlinearities are taken in account. The reinforcing steel bar is modelled as three-node beam element, and a two-node bond-link element of zero length is used for modelling the bond characteristic between the concrete and reinforcing steel bar in fire (see Fig. 1(b)). In order to consider the temperature distribution over the slab cross-section, the plain concrete elements are divided into layers, and each layer can have a different but uniform temperature at each time step.

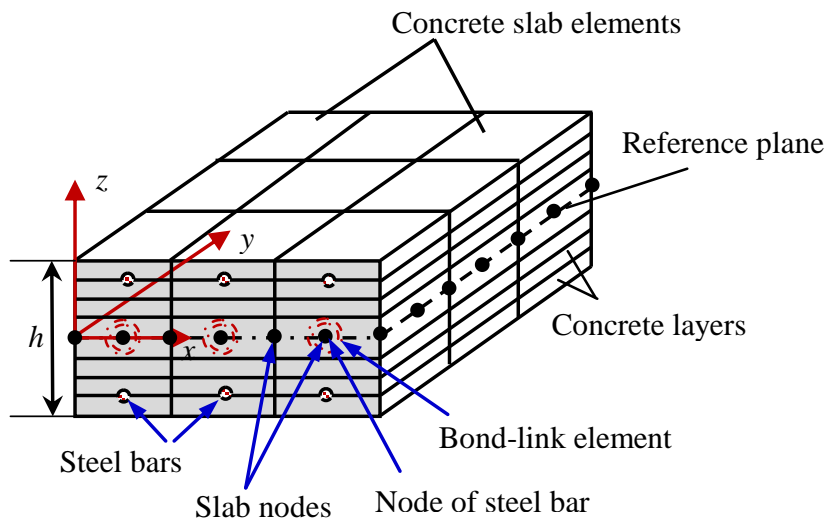

(a) Layered reinforced concrete slab model

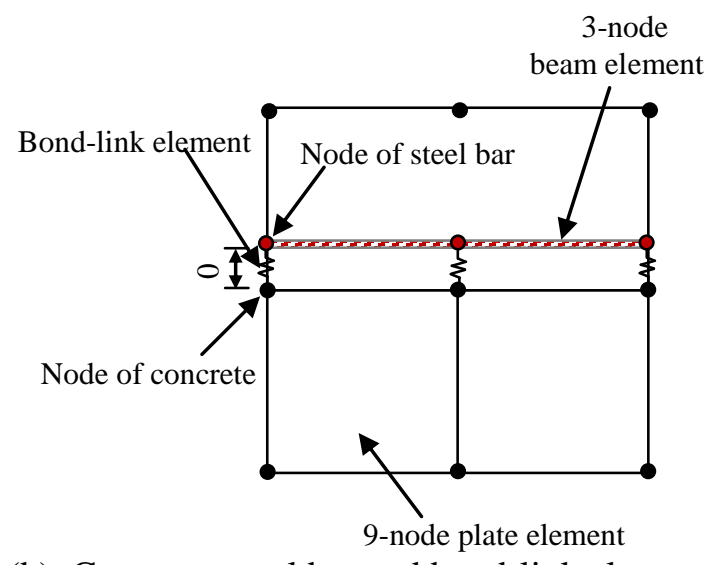

(b) Concrete, steel bar and bond-link element

Fig. 1 Finite element model of reinforced concrete slab

The nonlinear procedure presented in this paper is a hybrid finite element procedure combining both the smeared crack model, and the crack model with XFEM strong discontinuity. At the beginning of the analysis, the smeared crack model is used for modelling early stages of crack evolution, allowing the cracks to form layer by layer through the thickness of the plain concrete slab elements. Later, when the slab elements are subjected to large deformation, the 'delayed extended finite element method' (D-XFEM) is adopted for capturing individual large through-the-thickness cracks within the plain concrete elements. The detailed formulations of smeared crack model can be found in (Huang et al., 2003).

\subsection{Layered Concrete Elements with Delayed Extended Finite Element Model}

In this research, the delayed extended finite element method (D-XFEM) is incorporated into the plain concrete slab element for modelling individual big cracks when reinforced concrete slabs are subjected to large deformation in fire. In order to model the strong discontinuity within the concrete element, the partition of unity is adopted and the displacement field is then approximated by the sum of the regular displacement field and the enrichment displacement field (Moës et al., 1999). Considering a 9-node quadrilateral element crossed by a discontinuity $\left(\Gamma_{d}\right)$ the domain of an element is divided into two distinct domains, which are represented as $\Omega^{+}$and $\Omega^{-}$on the different sides of the discontinuity. The displacement field $\mathbf{u}$ consists of a continuous regular displacement field $\mathbf{u}_{\text {cont }}$ and a discontinuous displacement field $\mathbf{u}_{\text {dis }}$ :

$$
\mathbf{u}=\mathbf{u}_{\text {cont }}+\mathbf{u}_{\mathrm{dis}}=\sum_{1}^{9} N_{i} \mathbf{u}_{i}+\sum_{1}^{9} N_{i} \Psi_{i}(\mathbf{x}) \mathbf{a}_{i}
$$

where $N_{i}$ is the shape function, $\mathbf{u}_{i}$ is the regular node displacement, $\mathbf{a}_{i}$ is the additional node displacement to describe the discontinuity, and $\Psi_{i}(\mathbf{x})$ is the enrichment function:

$$
\Psi_{i}(\mathbf{x})=\operatorname{sign}(x)-\operatorname{sign}\left(x_{\mathrm{i}}\right) \quad(i=1 \sim 9)
$$

in which sign is the sign function and defined as:

$$
\operatorname{sign}(x)= \begin{cases}+1 & \text { if } x \in \Omega^{+} \\ -1 & \text { if } x \in \Omega^{-}\end{cases}
$$


For each node of the slab element there are five degrees of freedom (DOFs): three translational degrees of freedom $u, v, w$, and two rotational degrees of freedom $\theta_{x}, \theta_{y}$, where $x, y, z$ are local coordinates. Since the cracking model presented here is mainly intended to model the in-plane opening of individual cracks, rather than the strong discontinuity in rotations, only two membrane translational DOFs $(u, v)$ are enriched and all other DOFs $\left(w, \theta_{x}, \theta_{y}\right)$ remain as regular. Hence, only two additional DOFs $\left(u_{i}^{a}, v_{i}^{a}\right)$ per node are added to the nodal displacement vector, then node displacements $\mathbf{u}_{i}$ and $\mathbf{a}_{i}$ can be represented as:

$$
\begin{aligned}
& \mathbf{u}_{i}=\left[u_{1}^{u} v_{1}^{u} w_{1}^{u} \theta_{x 1}^{u} \theta_{y 1}^{u} u_{2}^{u} v_{2}^{u} w_{2}^{u} \theta_{x 2}^{u} \theta_{y 2}^{u} \ldots u_{9}^{u} v_{9}^{u} w_{9}^{u} \theta_{x 9}^{u} \theta_{y 9}^{u}\right]^{T} \\
& \mathbf{a}_{i}=\left[\begin{array}{llllll}
u_{1}^{a} & v_{1}^{a} & u_{2}^{a} v_{2}^{a} & \ldots & u_{9}^{a} & v_{9}^{a}
\end{array}\right]^{T}
\end{aligned}
$$

where $u_{i}^{u}$ and $v_{i}^{u}$ are the regular in-plane nodal displacements, $w_{i}^{u}$ is the regular out-plane nodal displacements, $\theta_{x i}^{u}$ and $\theta_{y i}^{u}$ are the regular nodal rotations, $u_{i}^{a}$ and $v_{i}^{a}$ are the enriched in-plane nodal displacements, respectively.

When a discontinuity is introduced in the element, the plate displacements can be expressed as:

$$
\left\{\begin{array}{l}
u=-\beta_{x} z+\left(u_{0}^{u}+u_{0}^{a}\right) \\
v=-\beta_{y} z+\left(v_{0}^{u}+v_{0}^{a}\right) \\
w=w_{0}
\end{array}\right.
$$

where $u_{0}^{u}$ and $v_{0}^{u}$ are regular in-plane displacements and $u_{0}^{a}, v_{0}^{a}$ are enriched in-plane displacements, respectively. $\beta_{x}$ and $\beta_{y}$ are rotations with respect to $x$ and $y$ axes, respectively, and $w_{0}$ is out-plane displacement along the $z$ coordinate.

To form the element stiffness matrix and internal force vector, the virtual work equation without body force reads as:

$$
\boldsymbol{\psi}(\mathbf{u})=\int_{\mathrm{V}} \overline{\mathbf{B}}^{\mathrm{T}} \boldsymbol{\sigma} d V-\mathbf{f}^{\mathrm{ext}}=\mathbf{0}
$$

where $\psi$ represents the sum of external and internal generalised forces, and in which $\overline{\mathbf{B}}$ is the strain-displacement transformation matrix. When introducing the XFEM formulations to Eq. (7), the regular internal force $\left(\mathbf{Q}^{u}\right)$ should balance the external force $\left(\mathbf{f}^{\mathrm{ext}}\right)$, and the enriched internal force $\left(\mathbf{Q}^{a}\right)$ must be zero, that is:

$$
\begin{aligned}
& \mathbf{Q}^{u}=\int_{\Omega} \mathbf{B}^{u^{T}} \boldsymbol{\sigma} d \Omega=\mathbf{f}^{\mathrm{ext}} \\
& \mathbf{Q}^{a}=\int_{\Omega^{+}, \Omega^{-}} \mathbf{B}^{a^{T}} \boldsymbol{\sigma} d \Omega=\mathbf{0}
\end{aligned}
$$

Using the Principle of Virtual Work on Eqs (8) and (9), the element stiffness matrix in terms of incremental displacements can be obtained as:

$$
\mathbf{K d} \mathbf{d}=\left[\begin{array}{ll}
\mathbf{K}^{u u} & \mathbf{K}^{u a} \\
\mathbf{K}^{a u} & \mathbf{K}^{a a}
\end{array}\right]\left\{\begin{array}{l}
\mathbf{d} \mathbf{u}_{i} \\
\mathbf{d \mathbf { a } _ { i }}
\end{array}\right\}=\left\{\begin{array}{l}
\mathbf{f}^{\mathrm{ext}} \\
0
\end{array}\right\}-\left\{\begin{array}{l}
\mathbf{Q}^{u} \\
\mathbf{Q}^{a}
\end{array}\right\}
$$

where $\mathbf{K}^{u u}$ is the element stiffness matrix referenced to regular DOFs, $\mathbf{K}^{a a}$ is the element stiffness matrix referenced to enriched DOFs, and $\mathbf{K}^{u a}=\left(\mathbf{K}^{a u}\right)^{T}$ is related to both. The details expressions of element stiffness matrix $\mathbf{K}^{u u}, \mathbf{K}^{u a}, \mathbf{K}^{a u}, \mathbf{K}^{a a}$ and internal force $\mathbf{Q}^{u}$ and $\mathbf{Q}^{a}$ can be found in (Liao and Huang, 2015).

In order to combine both smeared crack model and D-XFEM model into a hybrid finite element procedure, a criterion for determining the shift from the smeared crack model to extended finite element model needs to be proposed. Therefore in the current procedure, it is defined that a certain element will be enriched when all integration points over all layers of a slab element are failed by 
cracking based on the smeared crack model. For the enriched element, the extended finite element formulations will be introduced to calculate the element stiffness and internal force of the element. In this research, it is reasonable to assume that when a crack evolves layer by layer through the thickness of slab, the crack orientation generally keeps unchanged and is consistence with the initial crack orientation of the bottom layer. Therefore, for the enriched element the cracking orientation at the central fifth integration point (of the bottom layer of the element, predicted by the smeared crack model) is recorded and then used to determine the orientation of extended strong discontinuity for that enriched element. In this way, the individual large through-the-thickness crack within that enriched element is identified. Regarding the integration scheme, 9 Gauss integration points are employed to calculate the element stiffness matrix for the element with the smeared crack model. For the enriched elements, the scheme that partitions the element into 8 sub-triangles is adopted, where 7 Gauss points are applied to each sub-triangle and then totally 56 Gauss points are applied for an enriched element. The detailed work procedure of the current model was described in (Liao and Huang, 2015). The developments described above have been incorporated into software Vulcan (Huang et al., 2003, 2009) for modelling individual large cracks within the reinforced concrete slabs under fire conditions.

\subsection{Reinforcing Steel Bar and Bond-link Elements}

In the current model, the general 3D three-node beam element developed by the second author (Huang et al., 2009) is adopted to model the reinforcing steel bar. A bond-link element developed previously by (Huang, 2010) is employed to link the nodes between a plain concrete element and steel bar element. The characteristics of bond-link element are temperature dependent. Due to the highly non-linearity of the current model, a full Newton-Raphon solution procedure with load control is adopted.

\section{VALIDATIONS AND ANALYSIS}

In order to validate the present model, four reinforced concrete slabs tested in fire by (Bailey and Toh, 2007) were modelled for the validations. Due to the limit space, only tested slabs MF1 and MF2 are presented in this paper. The predicted load-central deflection curves of the slabs MF1 and MF2, together with the tested results, are shown in Fig. 2(a) and (b). It can be seen that generally, the predictions of the current model agree well with the test results. Compared with the predictions of the smeared crack model, the current model provides better predictions. The predicted cracking patterns of the slabs MF1 and MF2 are presented in Fig. 3(a) and (b), respectively. It can be seen that the predicted cracking patterns are generally close to those of the tested results. It is evident that the current model is capable to predict the initiation and propagation of the individual large cracks within a reinforced concrete slab.

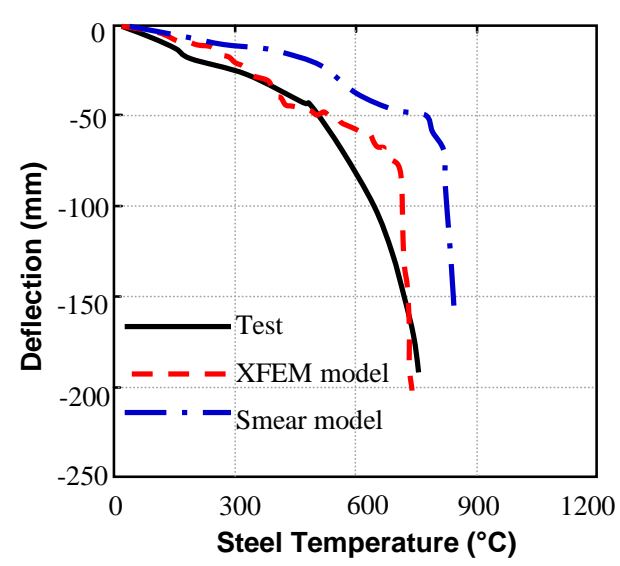

(a) Slab M1

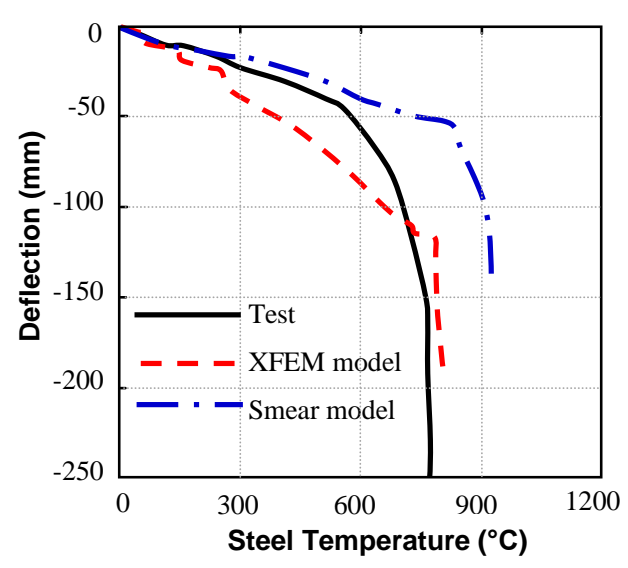

(b) Slab M2

Fig. 2 Comparison of predicted and tested maximum deflections 


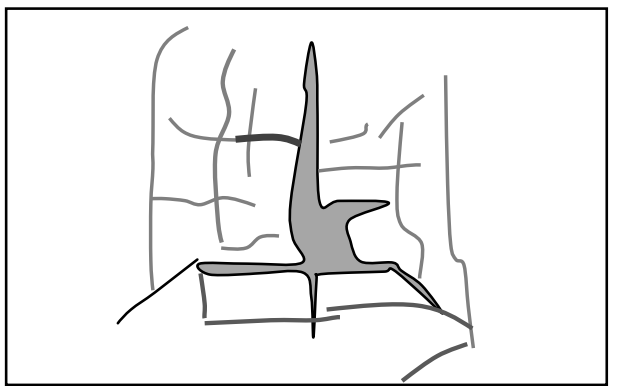

(a) Tested

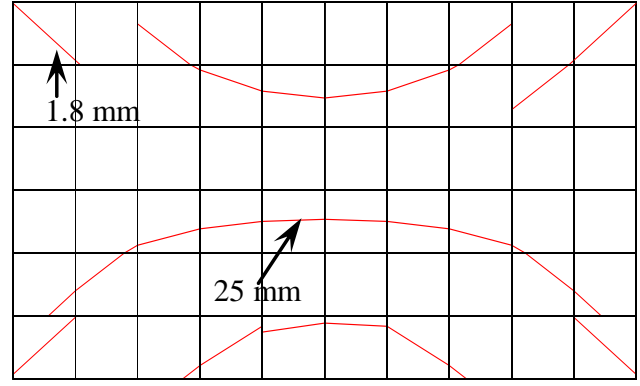

(b) Predicted

Fig. 3 Comparison of predicted and tested cracking pattern

In order to investigate the influence of supported steel beams on the crack propagations of composite floor under ISO834 fire, a series of parametric studies have been conducted by using the validated model. The modelled composite floor is a rectangular reinforced concrete slab supported by four structural steel beams on the edges. The overall dimensions of slab are $6000 \mathrm{~mm}$ in long span, $4000 \mathrm{~mm}$ in short span and $130 \mathrm{~mm}$ in thickness. More details of predicted composite floor can be found in (Liao and Huang, 2015). Fig. 4 presents the predicted temperatures used for the modelling. In this study the different protection conditions used for the steel beam a, b, c and $\mathrm{d}$ are: protecting all four beams (beam a, b, c, d); protecting three beams (beam b, c, d) only ;protecting two beams (beam $b, d$ ); protecting two beams (beam b, c); protecting one beam (beam c); no beams being protected. Fig. 5 presents the comparison of the maximum deflections for six different protection regimes. It can be seen that the deflection increases significantly when the number of protected beams is reduced. In the case of all four steel beams protected, the fire resistance of composite floor was about $120 \mathrm{~min}$, whilst the fire resistance decreased to $87.5 \mathrm{~min}$ for the case of three beams protected. When the number of protected beams was further reduced to two, the composite floor only had fire resistance of $32.5 \mathrm{~min}$ and $42.5 \mathrm{~min}$, respectively.
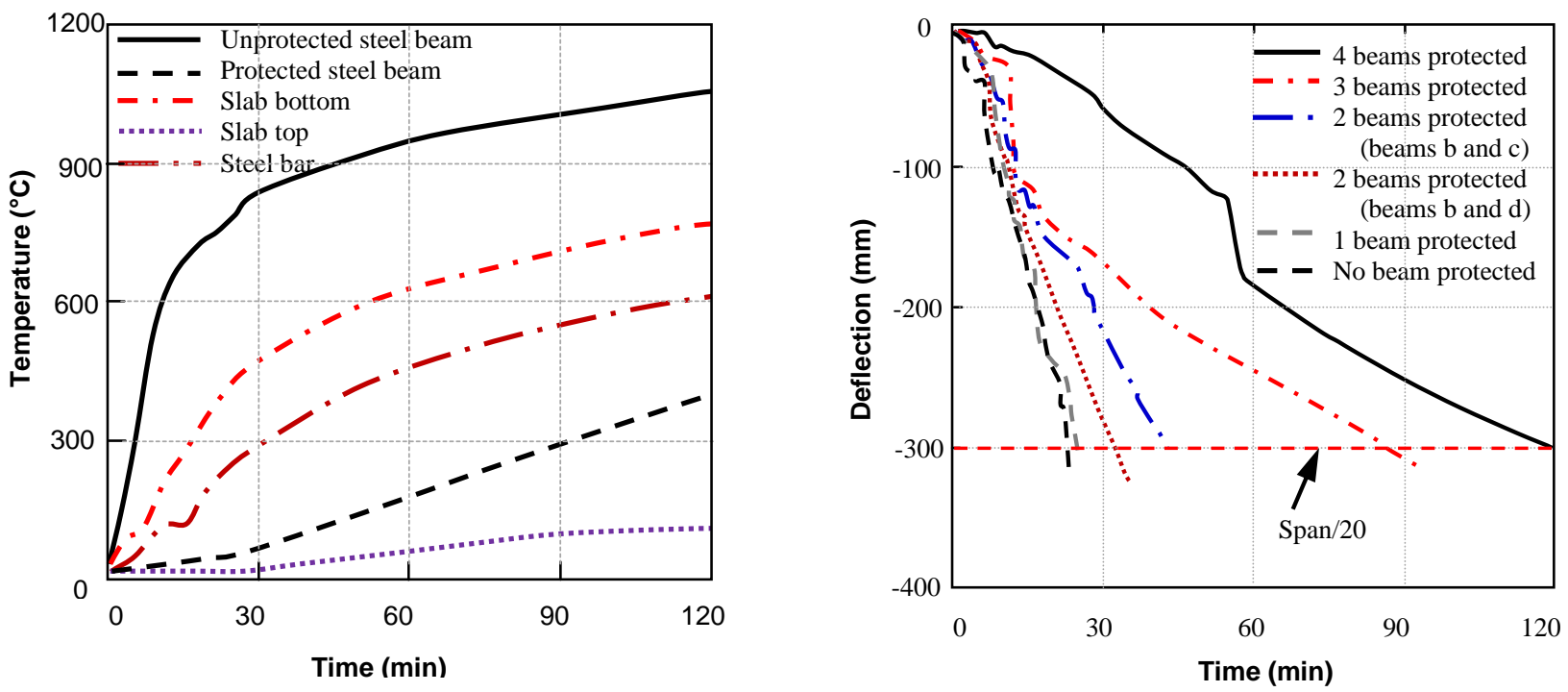

Fig. 4 Predicted temperatures

Fig. 5 Comparison of predicted maximum deflections

Due to the limit space, only two protection cases are presented here. As shown in Fig. 6(a), in the case of all four steel beams protected, two horizontal cracks and two vertical individual cracks appeared at the central zone of slab. Generally, the slab showed relatively good integrity. Fig. 6(b) shows the case with only two opposite short span beams (beams b and d) were protected, the slab can deform significantly along the long span and behave like a simply supported beam in fire. Therefore, cracks vertically crossed through the whole slab. In this case the membrane action was reduced significantly because the lack of the vertical support on the edges of the slab. Hence, the floor has a low ultimate load capacity in fire and is more likely to suffer from integrity failure. 


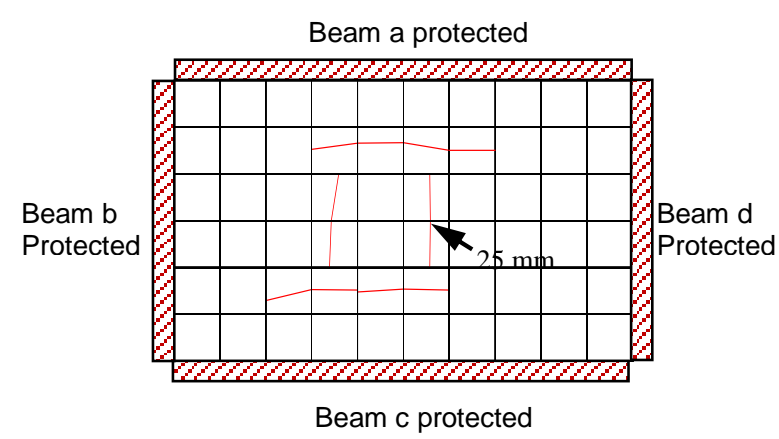

(a) 4 beams protected

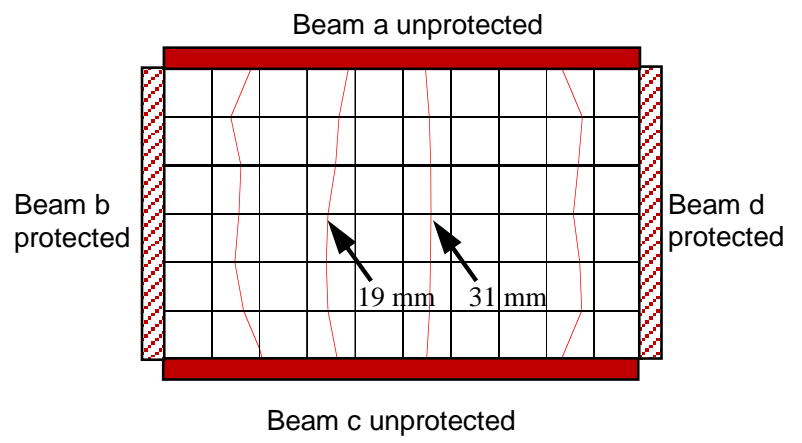

(b) 2 beams protected

Fig. 6 Comparison of predicted crack patterns of composite floor

\section{CONCLUSIONS}

In this paper a robust hybrid layered finite element procedure has been presented for modelling the initiation and propagation of individual large through-the-thickness cracks within the reinforced concrete slabs under fire conditions. For modelling concrete cracking, a hybrid model combined smeared crack model and extended strong discontinuity has been developed. In this new model the smeared crack approach is used for modelling early stages of crack evolution, and the 'delayed extended finite element method (D-XFEM)' is used for capturing individual big cracks within the reinforced concrete slabs when the slabs are subjected to large deformations. A robust criterion has been proposed for determining the shift from smeared crack model to extended finite element formulations.

This hybrid nonlinear procedure has been validated successfully for simulating the simply supported reinforced concrete slabs at elevated temperatures against the previous fire test results. The numerical studies performed on a composite floor slab with different protection regimes on the four edges' support beams indicated that the protection conditions of the edges' support beams have significant impacts on both global responses and cracking patterns of the composite floor in fire. Less protection for the edges' beams will damage the membrane action of concrete slab and result to integrity failure of the floor. The hybrid procedure developed in this paper is a very useful tool for assessing both structural stability and integrity of reinforced concrete or composite floor slabs under fire conditions.

\section{ACKNOWLEDGMENTS}

The authors gratefully acknowledge the support of the Engineering and Physical Sciences Research Council of Great Britain under Grant No. EP/I031553/1.

\section{REFERENCES}

Bailey CG., Toh WS. 2007. Small-scale concrete slab tests at ambient and elevated temperatures. Engineering Structures, 29(10), p. 2775-2791.

Huang Z., Burgess IW., Plank RJ. 2003. Modelling membrane action of concrete slabs in composite buildings in fire. Part I: Theoretical development. Journal of Structural Engineering, ASCE, 129(8), p.1093-1102.

Huang Z., Burgess IW., Plank RJ. 2009. Three-dimensional analysis of reinforced concrete beam-column structures in fire. Journal of Structural Engineering, ASCE, 135(10), p.1201-1212.

Huang Z. 2010. Modelling the bond between concrete and reinforcing steel in a fire. Engineering Structures, 32(11), p. 3660-3669.

Liao FY., Huang ZH. 2015. Modelling Integrity Failure of Reinforced Concrete Slabs under Fire Conditions. Report No. CEDPS-DMAC-2015-Fire-3, Brunel University.

Moës N., Dolbow J., Belytschko T. 1999. A finite element method for crack growth without remeshing. International Journal for Numerical Methods in Engineering, 46(1), p. 131-150.

$\mathrm{Xu}$ J., Lee CK., Tan KH. 2013. An XFEM frame for plate elements in yield line analyses. International Journal for Numerical Methods in Engineering, 96(3), p. 150-175. 